\section{(A) OPEN ACCESS}

\title{
Demographic and disease-related predictors of abnormal lung function in patients with established inflammatory polyarthritis and a comparison with the general population
}

\author{
Suzanne M M Verstappen, ${ }^{1}$ Mark Lunt, ${ }^{1}$ Robert N Luben, ${ }^{2}$ Jackie Chipping, ${ }^{3}$ \\ Tarnya Marshall, ${ }^{3}$ Kay-Tee Khaw, ${ }^{2}$ Nick Wareham, ${ }^{2}$ William G Dixon, ${ }^{1}$ lan N Bruce, ${ }^{1}$ \\ Deborah P M Symmons ${ }^{1,4}$
}

\begin{abstract}
Handling editor Tore K Kvien
- Additional data are published online only. To view this file please visit the journal online (http://dx.doi.org/10. 1136/annrheumdis-2012201698).

${ }^{1}$ Arthritis Research UK Epidemiology Unit, Manchester Academic Health Science Centre, The University of Manchester, Manchester, UK ${ }^{2}$ Department of Public Health and Primary Care, University of Cambridge School of Clinical Medicine, Cambridge, UK

${ }^{3}$ Department of Rheumatology, Norfolk and Norwich University Hospital, Norwich, UK ${ }^{4}$ NIHR Manchester Musculoskeletal Biomedical Research Unit, Manchester, UK
\end{abstract}

\section{Correspondence to}

Dr Suzanne M M Verstappen, Arthritis Research UK Epidemiology Unit, Manchester Academic Health Science Centre, University of

Manchester, Stopford Building, Oxford Road, Manchester M13 9PT, UK; Suzanne. Verstappen@manchester.ac.uk

Received 17 March 2012 Accepted 11 September 2012 Published Online First

13 October 2012

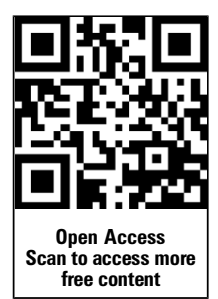

\section{ABSTRACT}

Objectives To identify demographic and clinical predictors of obstructive lung disease (OLD) and restrictive lung disease $(R L D)$ in patients with established inflammatory polyarthritis $(\mathrm{IP})$ and to compare the prevalence of respiratory symptoms in patients with IP and the general population.

Method A total of 421 patients with IP underwent a spirometry test 15 years after inclusion in the Norfolk Arthritis Register (NOAR). Logistic regression analyses were performed to assess the predictive ability of demographic and clinical characteristics obtained at inclusion in NOAR and to assess their association with OLD or RLD at 15 years (age- and gender-adjusted). In addition, the prevalence of OLD and RLD was compared with a matched population (1:4) of people participating in the European Prospective Investigation of Cancer-Norfolk, a representative sample of the general population in Norfolk, UK.

Results In this IP population, current smoking was the strongest predictor for OLD and functional disability for RLD. In the comparison study, $11.6 \%$ had OLD in the IP population and $4.9 \%$ in the general population (adjOR $2.01,95 \% \mathrm{Cl} 1.26$ to 3.22). The prevalence of RLD was not statistically different between the IP population and the general population (14.6\% vs $17.5 \%$; adjOR 0.76 , $95 \% \mathrm{Cl} 0.53$ to 1.10 ).

Conclusions OLD, but not RLD, is more prevalent in the IP population than in the general population. Functional disability is especially associated with RLD whereas smoking is associated with OLD. The latter finding, and the known association between smoking and a poor disease prognosis, underlines the importance of smoking cessation in patients with IP.

\section{INTRODUCTION}

Respiratory manifestations in rheumatoid arthritis (RA) are common and include upper and lower airway disorders, pleural and interstitial disorders including RA-associated interstitial lung disease (RA-ILD) and are associated with increased mortality. ${ }^{1-7}$ Common lung disorders can be divided into obstructive lung disease (OLD), including chronic obstructive bronchitis and emphysema, and restrictive lung disease (RLD), including RA-ILD. Especially for RLD, prevalence rates vary widely ranging from $1 \%$ to $50 \%$, depending on the diagnostic tests used (eg, pulmonary function tests, high-resolution CT (HRCT)) and the population studied. ${ }^{1}$ As there is an increased prevalence of smoking in patients with RA, it is perhaps not surprising that there should also be an increased prevalence of chronic obstructive pulmonary disease, lung cancer and respiratory infections compared with the general population. However, even after adjustment for smoking, increased rates of respiratory symptoms have been found in patients with RA compared with the general population. ${ }^{89}$

Several demographic and disease severity measures such as male gender, age, smoking, body mass index (BMI), erythrocyte sedimentation rate, rheumatoid factor (RF), anti-citrullinated protein antibody (ACPA) and human leucocyte antigen (HLA)-B40 have been identified as predictors of lung disease in patients with $\mathrm{RA}^{2}{ }^{10-13}$ although results differ between studies and sample sizes are relatively small. Moreover, most studies were restricted to investigating the prevalence and predictors of either OLD or RLD but not of both. Another disadvantage of previous publications is the cross-sectional study design. For some associations observed, such as between decreased functional disability and OLD or $\mathrm{RLD}$, it is not possible to establish the direction of the association. Therefore, it is also important to determine the association between potential predictors measured prior to the pulmonary function test and subsequent lung function disorders.

The objectives of this study were (1) to identify potential demographic and clinical predictors measured 15 years prior to and potential variables associated with OLD and RLD at time of the spirometry test in patients with inflammatory polyarthritis (IP), and (2) to compare the prevalence of abnormal lung function in patients with IP and the general population.

\section{METHODS}

\section{Clinical assessments}

Consecutive patients aged over 16 years with early IP from the Norfolk Arthritis Register (NOAR) recruited between 1990 and 1994 were included in this study. Details of NOAR have been published previously. ${ }^{14}$ Briefly, patients with swelling in two 
or more joints that lasted $\geq 4$ weeks were referred to NOAR. At baseline, patients were seen by a research nurse. Clinical assessments included the evaluation of the number of swollen and tender joints (out of a total of 51). Blood was collected and stored in $-80^{\circ} \mathrm{C}$ freezers to measure RF (positive $>40 \mathrm{Ul}$ ), ACPA $(\geq 5 \mathrm{U} / \mathrm{ml}$; Axis-Shield DIASTAT Kit, Axis-Shield, Dundee, UK) and C-reactive protein (CRP, mg/l). HLA genotyping was carried out as described previously and subtyping at the HLA-DRB1 locus was performed to identify the presence of the shared epitope. ${ }^{15}$ The 28-joint Disease Activity Score, based on three components including C-reactive protein (DAS28(3) $\mathrm{CRP})^{16}$ was calculated for each patient. In addition, patients completed the British version of the Health Assessment Questionnaire (HAQ). ${ }^{17}$ At each follow-up visit, smoking status was recorded and categorised into never smoked/past smoker/current smoker at baseline and 15-year follow-up. Clinical follow-up assessments were carried out annually for 3 years and then at 5, 7, 10, 12 and 15 years. RA was defined according to the 1987 American College of Rheumatology (ACR) criteria $^{18}$ and applied cumulatively over these years. At each assessment the use of disease-modifying antirheumatic drugs (DMARDs) and other medication, including medication for respiratory diseases (see online supplemental file), were also recorded. In NOAR, all patients remain under long-term follow-up except for (1) patients who do not cumulatively satisfy the ACR criteria for RA at 5 years and have been given a consultant diagnosis other than RA, undifferentiated IP, psoriatic arthritis or post-viral arthritis to explain their symptoms; (2) patients whose disease has gone into spontaneous long-term remission (no inflamed joints at the 3rd or 5th anniversary and not on DMARDs or steroids).

\section{Respiratory function in patients with IP}

A respiratory function test was performed at the 15-year assessment by a research nurse using a portable spirometer (Micro Medical GP MS07). A standardised protocol was used. Exclusion criteria were patients with angina, undiagnosed chest pains, cardiac problems, recent abdominal or eye surgery or frailty. Forced expiratory volume in $1 \mathrm{~s}\left(\mathrm{FEV}_{1}\right)$, percentage predicted $\mathrm{FEV}_{1}\left(\mathrm{FEV}_{1}\right.$ pred), forced vital capacity (FVC) and percentage predicted FVC (FVCpred) were recorded. OLD was defined according to British Thoracic Society criteria (ie, $\mathrm{FEV}_{1} / \mathrm{FVC}<0.70$ and FEV $_{1}$ pred $\left.<80 \%\right) .{ }^{19}$ RLD was defined as FVCpred $<70 \%$ and $\mathrm{FEV}_{1}$ /FVC between 0.80 and 1.00. Normal lung function was defined as FVCpred $\geq 70 \%$ and $\mathrm{FEV}_{1}$ pred $\geq 80 \%$. Patients also completed the Medical Research Council (MRC) respiratory symptom questionnaire. ${ }^{2021}$ The MRC dyspnoea scale consists of five statements about perceived breathlessness (see figure 1).

Patients with MRC grades 3, 4 or 5 were defined as having moderate to severe breathlessness and were compared with patients with MRC grades 1 or 2 .

\section{Respiratory function in the general population}

Respiratory data collected as part of the European Prospective Investigation of Cancer (EPIC-Norfolk) in Norfolk, UK were used as reference data for the general population. ${ }^{22} 23$ Participants in EPIC-Norfolk are men and women aged 39-79 years at inclusion into the cohort and resident in Norfolk, UK-that is, the same residential area as that of the patients in NOAR. Participants in EPIC-Norfolk were recruited between 1993 and 1997 and follow-up data have been collected at regular intervals. For the present study, data obtained during the second assessment (1998-2000) were used. Participants were asked to complete a questionnaire including questions about any medical conditions, medication use and smoking history (never, past or current). At a clinic visit, height and weight were measured and respiratory function was assessed by spirometry (Micro Medical, Rochester, UK). FEV 1 and FVC were measured twice and the higher measure was recorded. Participants in EPIC-Norfolk who were also included in NOAR, were using DMARDs, had a diagnosis of RA, had incomplete respiratory data or missing data on height were excluded from the general population cohort in this study.

\section{Comparison of respiratory function between patients with IP and the general population}

Since the age range between patients with IP and the general population sample was slightly different and age is strongly associated with lung function, we included only those people aged between 42 and 81 years at the time the respiratory test was performed. Patients with IP were matched 1-4 with people from the general population by age, gender and season in which the spirometry test was performed. By season and gender, controls were matched using a greedy algorithm to select age matches by selecting the closest match first, within a 5 -year age range, then the closest remaining match and so on (STATA, gmatch). FEV 1 pred and FVCpred were not recorded in EPIC and therefore we estimated the expected $\mathrm{FEV}_{1}$ and FVC for both cohorts to calculate the percentage of predicted value (observed/estimated expected $\times 100 \%$ ). The estimated expected value was calculated based on the equation from the Health Survey for England. ${ }^{24}$ The estimated $\mathrm{FEV}_{1}$ pred and FVCpred values were strongly correlated with the actual recorded $\mathrm{FEV}_{1}$ pred and FVCpred values within the NOAR cohort (FEV 1 pred: $r=0.97, p<0.001$; FVCpred: $r=0.96, p<0.001$ ).

NOAR and EPIC-Norfolk were approved by the local Norwich Research Ethics Committee and all patients and participants gave written informed consent.

\section{Statistical analysis}

In the IP population the predictive ability of baseline demographic and disease-related characteristics and the association between demographic and disease related factors measured at

\begin{tabular}{|l|}
\hline Grade I: I only get breathless with strenuous exercise \\
\hline \hline Grade II: I get short of breath when hurrying on the level or up a slight hill exercise \\
\hline \hline $\begin{array}{l}\text { Grade III: I walk slower than people of the same age on the level because of } \\
\text { breathlessness or have to stop for breath when walking at my own pace on the level or } \\
\text { upa slight hill exercise. }\end{array}$ \\
\hline \hline Grade IV: I stop for breath after walking 100 yards or after a few minutes on the level \\
\hline \hline Grade V: I am too breathless to leave the house \\
\hline
\end{tabular}

Figure 1 Description grades of MRC respiratory symptom questionnaire. 
15 years with OLD or RLD at the 15 -year follow-up were assessed using logistic regression models. OR (95\% CI) were adjusted for age and gender (adjOR). For the comparison study between patients with IP and the general population, a multinomial regression analysis was performed to test the difference in smoking rates between patients with IP and the general population. A random linear or logistic effect model, where appropriate, retaining the matched groups, was applied to test the difference in $\mathrm{FEV}_{1}$ and FVC between patients with IP and the general population and to test the likelihood for OLD and RLD adjusting for smoking status (adj $\beta$ or adjOR). All analyses were repeated in the group of patients fulfilling the 1987 ACR criteria for RA.

All analyses were carried out using STATA Release 11 (StataCorp, College Station, Texas, USA).

\section{RESULTS}

A total of 1098 eligible patients were recruited by NOAR between 1990 and 1994; 304 died before the expected 15-year follow-up assessment date, in $12 \%$ of which the underlying cause of death was respiratory disease (ICD-10 code $\mathrm{J}^{*}$, see online supplement). In addition, 271 patients were lost to follow-up or were not followed after the 5-year assessment as indicated by the protocol. A total of 523 patients were seen for their 15-year assessment. Of these, 102 patients fulfilled the exclusion criteria or did not want to perform the spirometry test, leaving 421 patients with complete respiratory data included in this study.

At 15 years the mean (SD) age of these 421 patients was 62 (13) years and mean disease duration since symptom onset was 16.0 (1.1) years; 68\% were female (table 1) and 79.3\% cumulatively fulfilled the 1987 ACR criteria for RA. Disease activity was moderate with a median (IOR) DAS28 score of 3.0 (2.2-3.9) and a median HAQ score of 0.88 (0.25-1.63). Thirty-nine percent of the patients were using non-biological DMARDs and about $6 \%$ used a biological agent.

\section{Abnormal lung function test}

Mean (SD) $\mathrm{FEV}_{1}$ and FVC were slightly higher for men than for women (2.65 (0.77) 1 vs $2.10(0.60) 1$ and 3.39 (0.94) 1 vs $2.62(0.73) 1$, respectively). $6.1 \%$ of female patients and $17.1 \%$ of male patients satisfied the criteria for OLD and $6.9 \%$ of female patients and $24.7 \%$ of male patients satisfied the criteria for RLD. Forty-seven of 421 patients (11\%) were not categorised as having OLD, RLD or normal lung function. In the total IP population, $12.4 \%$ used medication for respiratory symptoms. Of the 419 patients who completed the MRC respiratory questionnaire, $14.1 \%$ ( $14.7 \%$ of women and $12.8 \%$ of men) reported being breathless ( $\geq$ grade 3 ).

\section{Associations with OLD and RLD}

Table 2 shows the baseline predictors of abnormal lung function test at 15 years. Age and male gender were predictors of both OLD and RLD in patients with IP. Current smoking at baseline (adjOR 15.25, 95\% CI 3.14 to 73.99) and a trend towards previous smoking (adjOR 4.59, 95\% CI 0.99 to 21.33) were strong predictors of OLD. DAS28 measured at baseline (adjOR 1.41, 95\% CI 1.00 to 1.99) was also a predictor of OLD. No other clinical variables were associated with OLD, whereas RLD was predicted by a high baseline tender joint count (adjOR 1.07, 95\% CI 1.02 to 1.12), worse DAS28 score (adjOR $1.44,95 \%$ CI 1.09 to 1.91 ) and higher HAQ score (adjOR 2.28, $95 \%$ CI 1.36 to 3.83 ). In addition, patients who fulfilled the 1987 criteria for RA (adjOR 2.56, 95\% CI 1.24 to 5.29) and
Table 1 Demographic, clinical characteristics and spirometry values at 15 years

\begin{tabular}{|c|c|c|}
\hline Variable & $\mathbf{N}$ & \\
\hline \multicolumn{3}{|l|}{ Demographic characteristics } \\
\hline Age, years & 421 & $62(13)$ \\
\hline Gender, female & 421 & $288(68.4 \%)$ \\
\hline Disease duration, years & 415 & $16.0(1.1)$ \\
\hline BMI & 421 & $27(24-31)$ \\
\hline Smoking status & 421 & \\
\hline Never & & $141(33.5 \%)$ \\
\hline Past & & $217(51.5 \%)$ \\
\hline Current & & $63(15.0 \%)$ \\
\hline \multicolumn{3}{|l|}{ Clinical characteristics } \\
\hline Swollen joint count, 28-joint count & 421 & $0(0-3)$ \\
\hline Tender joint count, 28-joint count & 421 & $2(0-6)$ \\
\hline DAS28 ${ }_{\mathrm{CRP}(3)}$ & 325 & $3.0(2.2-3.9)$ \\
\hline $\mathrm{HAO}$ & 420 & $0.88(0.25-1.63)$ \\
\hline CRP, mg/l & 325 & $9.0(3.6-17.0)$ \\
\hline $\mathrm{RF}^{*}$, positive & 376 & $98(26.1 \%)$ \\
\hline ACPA* $^{*}$, positive & 323 & $74(22.9 \%)$ \\
\hline Shared epitope: & 392 & \\
\hline 0 allele & & $159(40.6 \%)$ \\
\hline 1 allele & & $170(43.4 \%)$ \\
\hline 2 alleles & & $63(16.1 \%)$ \\
\hline RA, 1987 criteria & 421 & $334(79.3 \%)$ \\
\hline Nodules & 421 & $44(10.5 \%)$ \\
\hline \multicolumn{3}{|l|}{ Medication use } \\
\hline nbDMARD & 421 & $166(39.4 \%)$ \\
\hline Biologics & 421 & $26(6.2 \%)$ \\
\hline Oral steroids & 421 & $47(11.2 \%)$ \\
\hline \multicolumn{3}{|l|}{ Abnormal lung function test } \\
\hline $\mathrm{FEV}_{1}$ & 421 & $2.28(0.70)$ \\
\hline Men & & $2.65(0.77)$ \\
\hline Women & & $2.10(0.60)$ \\
\hline FVC & 421 & $2.86(0.88)$ \\
\hline Men & & $3.39(0.94)$ \\
\hline Women & & $2.62(0.73)$ \\
\hline Obstructive lung disease $†$ & 333 & $30(9.0 \%)$ \\
\hline Men & & $15(17.1 \%)$ \\
\hline Women & & $15(6.1 \%)$ \\
\hline Restrictive lung disease $\dagger$ & 344 & $41(11.9 \%)$ \\
\hline Men & & $24(24.7 \%)$ \\
\hline Women & & $17(6.9 \%)$ \\
\hline Medication for respiratory symptoms & 421 & $52(12.4 \%)$ \\
\hline Breathlessness & 419 & $59(14.1 \%)$ \\
\hline
\end{tabular}

$\mathrm{N}=$ number of patients with available data. Values are mean (SD), median (IQR) for continuous variables or $\mathrm{n}(\%)$ for categorical variables.

${ }^{*}$ Measured at baseline.

tCompared with patients with normal spirometry lung function test (see Methods section for description of obstructive, restrictive and normal spirometry lung function test).

ACPA, anti-citrullinated protein antibody; BMI, body mass index; CRP, C-reactive protein; DAS28, 28-joint Disease Activity Score; FEV ${ }_{1}$, forced expiratory volume in $1 \mathrm{~s} ; \mathrm{FVC}$, forced vital capacity; HAQ, Health Assessment Questionnaire; nbDMARD, non-biological disease-modifying antirheumatic drug; RA, rheumatoid arthritis; RF, rheumatoid factor.

patients with nodules (adjOR 4.79, 95\% CI 1.46 to 15.71) at baseline were more likely to have RLD 15 years after inclusion in the NOAR cohort. To further test the association between clinical and demographic characteristics measured early in the disease with the development of lung disease, the analyses were repeated excluding patients $(n=12)$ who used drugs for respiratory symptoms at baseline since we did not perform a 
Table 2 Baseline predictors of obstructive and restrictive lung disease

\begin{tabular}{|c|c|c|c|c|c|c|c|c|}
\hline & \multirow[b]{2}{*}{$\mathrm{N}=303$} & \multirow[b]{2}{*}{ Normal } & \multicolumn{3}{|c|}{ Obstructive lung disease } & \multicolumn{3}{|c|}{ Restrictive lung disease } \\
\hline & & & $\mathbf{N}$ & Obstructive $\mathbf{N}=\mathbf{3 0}$ & OR (95\% Cl) & $\mathbf{N}$ & Restrictive $\mathrm{N}=41$ & OR (95\% Cl) \\
\hline \multicolumn{9}{|l|}{ Demographic characteristics } \\
\hline Age, years & 303 & $44(13)$ & 30 & $53(9)$ & $1.06(1.02$ to 1.09$)$ & 41 & $51(13)$ & 1.04 (1.01 to 1.07$)$ \\
\hline Gender, female & 303 & $230(76 \%)$ & 30 & $15(50 \%)$ & $0.32(0.15$ to 0.68$)$ & 41 & $17(41 \%)$ & 0.22 (0.11 to 0.44 ) \\
\hline Smoking status & 303 & & 30 & & & 41 & & \\
\hline Never & & $112(37 \%)$ & & $2(7 \%)$ & 1 & & $13(32 \%)$ & 1 \\
\hline Past & & $123(41 \%)$ & & $14(47 \%)$ & 4.59 (0.99 to 21.33$)$ & & $16(39 \%)$ & $0.64(0.28$ to 150$)$ \\
\hline Current & & $68(22 \%)$ & & $14(47 \%)$ & 15.25 (3.14 to 73.99$)$ & & $12(29 \%)$ & 1.45 (0.59 to 3.56$)$ \\
\hline \multicolumn{9}{|l|}{ Clinical characteristics } \\
\hline Swollen joint count, 28 joints & 303 & $4(1-9)$ & 30 & $5(2-11)$ & 1.01 (0.96 to 1.08 ) & 41 & $6(3-14)$ & 1.05 (1.00 to 1.11$)$ \\
\hline Tender joint count, 28 joints & 303 & $5(2-10)$ & 30 & $4(1-15)$ & 1.04 (0.99 to 1.10$)$ & 41 & $7(3-13)$ & 1.07 (1.02 to 1.12 ) \\
\hline DAS28 ${ }_{\mathrm{CRP}(3)}$ & 251 & $3.70(2.75-4.60)$ & 22 & $4.12(3.53-4.88)$ & 1.41 (1.00 to 1.99$)$ & 33 & $4.10(3.35-5.68)$ & 1.44 (1.09 to 1.91$)$ \\
\hline $\mathrm{HAO}$ & 297 & $0.625(0.25-1.13)$ & 30 & $0.563(0.38-1.0)$ & 1.01 (0.55 to 1.87$)$ & 41 & $0.875(0.38-1.63)$ & 2.28 (1.36 to 3.83 ) \\
\hline CRP, mg/l & 251 & $3(0-9)$ & 22 & $4(8.5-13)$ & 1.00 (0.99 to 1.02$)$ & 33 & $9(2-17)$ & 1.00 (0.99 to 1.02 ) \\
\hline RF, positive & 274 & $64(23 \%)$ & 24 & $9(38 \%)$ & 2.27 (0.90 to 5.75$)$ & 36 & $12(33 \%)$ & 1.63 (0.74 to 3.58 ) \\
\hline ACPA, positive & 246 & $54(22 \%)$ & 17 & $3(18 \%)$ & 0.70 (0.19 to 2.60$)$ & 24 & $10(42 \%)$ & 2.25 (0.90 to 5.63$)$ \\
\hline Shared epitope & 283 & & 29 & & & 36 & & \\
\hline 0 allele & & $116(41 \%)$ & & $9(31 \%)$ & 1 & & $15(42 \%)$ & 1 \\
\hline 1 allele & & $122(43 \%)$ & & $14(48 \%)$ & 1.44 (0.58 to 3.57$)$ & & $15(42 \%)$ & 086 (0.39 to 1.93 ) \\
\hline 2 alleles & & $45(16 \%)$ & & $6(21 \%)$ & 1.79 (0.57 to 5.64$)$ & & $6(17 \%)$ & 0.96 (0.33 to 2.80$)$ \\
\hline RA, 1987 criteria & 303 & $121(40 \%)$ & 30 & $12(40 \%)$ & 0.94 (0.42 to 2.14$)$ & 41 & $24(59 \%)$ & 2.56 (1.24 to 5.29$)$ \\
\hline Nodules & 303 & $13(4 \%)$ & 30 & $3(10 \%)$ & $2.60(0.64$ to 10.59$)$ & 41 & $5(12 \%)$ & 4.79 (1.46 to 15.71$)$ \\
\hline
\end{tabular}

$\mathrm{N}=$ number of patients with available data. Values are mean (SD), median (IQR) for continuous variables or $\mathrm{n}(\%)$ for categorical variables.

See Methods section for description of obstructive, restrictive and normal spirometry lung function test.

Bold type indicates statistical significance $(p<0.05)$.

ACPA, anti-citrullinated protein antibody; CRP, C-reactive protein; DAS28, 28-joint Disease Activity Score; HAQ, Health Assessment Questionnaire; RA, rheumatoid arthritis; RF, rheumatoid factor; RLD, restrictive lung disease.

spirometry test at baseline and thus could not define patients as having OLD or RLD at baseline. DAS28 was no longer a predictor for OLD ( $p=0.062$ ), probably because of lack of power, whereas all other results remained the same.

Cross-sectional associations between demographic and clinical variables with OLD and RLD at 15 years are shown in table 3 . Age and male gender at the 15-year follow-up were associated with OLD and RLD. Current smoking at 15 years (adjOR 15.91, 95\% CI 3.00 to 84.3 ) and also previous smoking (adjOR 5.90, 95\% CI 1.32 to 26.4) were associated with OLD at 15 years. This could probably be explained by the 51 patients who were smoking at baseline and stopped smoking during follow-up. Among all the patients who stopped smoking prior to the 15 -year follow-up visit and with available data on time of smoking cessation $(n=148)$, the risk of having OLD reduced with longer cessation time (OR $0.98,95 \%$ CI 0.94 to 1.02 ) although this was not significant.

Patients with a high DAS28 were more likely to have OLD (adjOR 1.49, 95\% CI 1.05 to 2.10) but not RLD (adjOR 1.22, $95 \%$ CI 0.83 to 1.80 ). In contrast, patients with decreased functional ability were more likely to have RLD (adjOR $2.90,95 \% \mathrm{CI}$ 1.78 to 4.71 ) and a trend for OLD (adjOR $1.60,95 \%$ CI 0.96 to 2.66). High BMI (adjOR 1.08, 95\% CI 1.00 to 1.16 ) was associated with RLD and, although not significant, there was a trend towards an association between CRP (adjOR 1.01, 95\% CI 1.00 to 1.03 ), nodules (adjOR $2.22,95 \%$ CI 0.88 to 5.57 ) and RLD. Use of steroids at the time of the 15 -year assessment was associated with RLD, but use of methotrexate did not differ between patients with lung function abnormalities and those without. Restricting the analyses to patients who cumulatively fulfilled the 1987 ACR criteria for RA resulted in similar findings (see online supplementary file tables S1 and S2).

\section{Comparison of abnormal lung function test between patients with IP and the general population}

This part of the study included 373 patients with IP and 1492 people from the general population in Norfolk, UK (table 4). The mean (SD) age in both cohorts was 62 (11) years and $68 \%$ were women. Patients with IP were more often current smokers (relative risk ratio (RRR) $2.76,95 \%$ CI 1.89 to 4.02 ) or ex-smokers (RRR 1.97, 95\% CI 1.54 to 2.52 ) than people from the general population. Although a small difference, $\mathrm{FEV}_{1}$ was significantly lower for patients with IP than in the general population (adj $\beta-0.08,95 \% \mathrm{CI}-0.13$ to -0.02 ) but FVC was similar between the two populations $(\operatorname{adj} \beta$ 0.06, $95 \%$ CI -0.01 to 0.13 ) with adjustment for smoking. However, when adjusting for smoking and BMI, patients with IP were less likely to have RLD than the general population (table 4). Thirty-two of 277 patients $(11.6 \%)$ had OLD in the IP population compared with 55/1124 (4.9\%) in the general population (adjOR 2.01, 95\% CI 1.26 to 3.22); for women and men these figures were, respectively, 18/197 (9.1\%) vs 25/789 (3.2\%) (adjOR 2.47, 95\% CI 1.29 to 4.71 ) and $14 / 80$ (17.5\%) vs $30 / 335$ (9.0\%) (adjOR $1.76,95 \%$ CI 0.87 to 3.58 ). RLD was observed in $14.6 \%(42 / 287)$ of the IP population and in $17.5 \%$ (227/1296) of the general population (adjOR $0.76,95 \%$ CI 0.53 to 1.10). Eleven percent (22/201) women with IP and $13.4 \%(118 / 882)$ women in the general population had RLD (adjOR $0.77,95 \%$ CI 0.47 to 1.28 ). In men these percentages were $23.3 \%(20 / 86)$ and $26.3 \%$ (109/414), respectively (adjOR $0.82,95 \%$ CI 0.47 to 1.43 ). Overall, patients with IP used medication for respiratory symptoms more often than people from the general population (12.1\% vs $2.8 \%$ ) (OR $4.84,95 \%$ CI 2.91 to 8.06). 
Table 3 Associations of obstructive and restrictive lung disease: cross-sectional analysis at 15 years

\begin{tabular}{|c|c|c|c|c|c|c|c|c|}
\hline & \multirow[b]{2}{*}{$\mathbf{N}$} & \multirow[b]{2}{*}{ Normal } & \multicolumn{3}{|c|}{ Obstructive lung disease } & \multicolumn{3}{|c|}{ Restrictive lung disease } \\
\hline & & & $\mathbf{N}$ & Obstructive $\mathrm{N}=\mathbf{3 0}$ & OR $(95 \% \mathrm{Cl})$ & $\mathbf{N}$ & Restrictive $N=41$ & OR (95\% Cl) \\
\hline \multicolumn{9}{|l|}{ Demographic characteristics } \\
\hline Gender, female & 303 & $230(76 \%)$ & 30 & $15(50 \%)$ & $0.32(0.15$ to 0.68$)$ & 41 & $17(41 \%)$ & $0.22(0.11$ to 0.44$)$ \\
\hline BMI & 303 & $27.5(4.9)$ & 30 & $28.0(5.4)$ & $1.02(0.94$ to 1.10$)$ & 41 & $29.2(5.1)$ & $1.08(1.00$ to 1.16$)$ \\
\hline Smoking status & 303 & & 30 & & & 41 & & \\
\hline Current & & $37(12 \%)$ & & $8(27 \%)$ & $15.91(3.00$ to 84.3$)$ & & $9(22 \%)$ & 2.01 (0.74 to 5.50$)$ \\
\hline \multicolumn{9}{|l|}{ Clinical characteristics } \\
\hline Swollen joint count, 28 joints & 303 & $0(0-3)$ & 30 & $1(0-3)$ & $1.05(0.94$ to 1.17$)$ & 41 & $0(0$ to 3$)$ & 1.01 (0.91 to 1.13$)$ \\
\hline Tender joint count, 28 joints & 303 & $2(0-6)$ & 30 & $2(0-10)$ & $1.05(1.00$ to 1.11$)$ & 41 & $1(0$ to 7$)$ & 1.04 (0.99 to 1.09$)$ \\
\hline DAS28 ${ }_{\mathrm{CRP}(3)}$ & 237 & $2.97(2.18-3.80)$ & 28 & $3.46(2.32-4.43)$ & $1.49(1.05$ to 2.10$)$ & 24 & 3.05 (2.43 to 3.67$)$ & $1.22(0.83$ to 1.80$)$ \\
\hline Current Methotrexate use & 303 & $84(28 \%)$ & 30 & $7(23 \%)$ & $0.67(0.27$ to 1.68$)$ & 41 & $6(15 \%)$ & 0.42 (0.16 to 1.06$)$ \\
\hline Current oral steroids use & 303 & $23(8 \%)$ & 30 & $6(20 \%)$ & 2.23 (0.77 to 6.42$)$ & 41 & $10(24 \%)$ & $3.22(1.30$ to 7.98$)$ \\
\hline \multicolumn{9}{|l|}{ Respiratory symptoms } \\
\hline Medication for respiratory symptoms & 303 & $32(11 \%)$ & 30 & $8(27 \%)$ & $3.68(1.42$ to 9.55$)$ & 41 & $6(15 \%)$ & 1.66 (0.61 to 4.52$)$ \\
\hline Breathlessness & 301 & $33(11 \%)$ & 30 & $8(27 \%)$ & $2.80(1.09$ to 7.20$)$ & 41 & $9(22 \%)$ & 2.28 (0.93 to 5.58$)$ \\
\hline
\end{tabular}

$\mathrm{N}=$ number of patients with available data. Values are mean (SD), median (IOR) for continuous variables or $\mathrm{n}(\%)$ for categorical variables.

See Methods section for description of obstructive, restrictive and normal spirometry lung function test.

Bold type indicates statistical significance $(p<0.05)$.

BMI, body mass index; CRP, C-reactive protein; DAS28, 28-joint Disease Activity Score; HAQ, Health Assessment Questionnaire; RA, rheumatoid arthritis; RLD, restrictive lung disease.

\section{DISCUSSION}

In this study of patients with established IP, 9.0\% had OLD and $11.9 \%$ had RLD. The percentage with OLD was higher than in the general population matched for age and gender. We found a strong association between smoking and OLD, an association not observed with RLD. A number of measures of disease severity assessed early in the disease were mainly predictive of RLD after 15 years. However, DAS28 was the only clinical variable associated with OLD in both the longitudinal and the crosssectional analysis. Nodules, another extra-articular manifestation of RA, were also associated with RLD.

In previous studies inconsistent results have been found between measures of disease activity, demographic factors, smoking and RLD and OLD. ${ }^{2} 10-13$ The findings of our study are supported by prior studies that have identified clinical and other demographic factors in association with either RLD or

Table 4 Comparison of spirometry data for patients with IP and the general population

\begin{tabular}{|c|c|c|c|c|c|}
\hline Variable & $\mathbf{N} / \mathbf{n}$ & $\begin{array}{l}\text { Mean (SD) or n (\%) } \\
\text { General population } \\
(\mathrm{N}=1492)\end{array}$ & $\begin{array}{l}\text { Mean (SD) or n (\%) } \\
\text { IP patients } \\
(\mathrm{N}=373)\end{array}$ & $\begin{array}{l}\text { Difference: } \boldsymbol{\beta}(95 \% \mathrm{CI}) \\
\text { or OR }(95 \% \mathrm{CI}) \\
\text { Adjusted for } \\
\text { smoking }\end{array}$ & $\begin{array}{l}\text { Difference: } \boldsymbol{\beta}(95 \% \mathrm{CI}) \\
\text { or OR (95\% CI) } \\
\text { Adjusted for smoking } \\
\text { and BMI }\end{array}$ \\
\hline \multicolumn{6}{|l|}{ Demographic characteristics } \\
\hline Age, years & $1492 / 373$ & $62(11)$ & $62(11)$ & & \\
\hline Gender, female & $1492 / 373$ & $1012(68 \%)$ & $253(68 \%)$ & & \\
\hline \multicolumn{6}{|l|}{ Smoking status } \\
\hline Never & & 767 (51.4\%) & $125(33.5 \%)$ & & \\
\hline Past & & $607(40.7 \%)$ & $195(52.3 \%)$ & & \\
\hline Current & & $118(7.9 \%)$ & $53(14.2 \%)$ & & \\
\hline BMI, $\mathrm{kg} / \mathrm{m}^{2}$ & $1490 / 373$ & $26.6(4.1)$ & $27.9(5.0)$ & & \\
\hline \multicolumn{6}{|l|}{ Abnormal lung function test } \\
\hline Medication for respiratory symptoms & $1492 / 373$ & $41(2.8 \%)$ & $45(12.1 \%)$ & & \\
\hline $\mathrm{FEV}_{1}$ & $1492 / 373$ & $2.36(0.71)$ & $2.28(0.69)$ & $-0.08(-0.13$ to -0.02$)$ & $-0.06(-0.11$ to -0.01$)$ \\
\hline FVC & $1492 / 373$ & $2.82(0.87)$ & $2.88(0.86)$ & $0.06(-0.01$ to 0.13$)$ & $0.08(0.01$ to 0.15$)$ \\
\hline Obstructive lung disease & $1124 / 277$ & $55(4.9 \%)$ & $32(11.6 \%)$ & $2.01(1.26$ to 3.22$)$ & $2.05(1.27$ to 3.29$)$ \\
\hline Restrictive lung disease & $1296 / 287$ & $227(17.5 \%)$ & $42(14.6 \%)$ & $0.76(0.53$ to 1.10$)$ & 0.71 (0.49 to 1.03$)$ \\
\hline
\end{tabular}

$\mathrm{N}=$ general population $\mathrm{n}=$ patients with IP with available data.

$\mathrm{BMI}$, body mass index; $\mathrm{FEV}_{1}$, forced expiratory volume in $1 \mathrm{~s}$; FVC, forced vital capacity; IP, inflammatory polyarthritis. 
OLD with respect to $\mathrm{HAO}$, BMI, male gender and age. However, in contrast to other studies, ${ }^{10}$ we did not find a significant association between ACPA and RF and lung function abnormalities, but a trend between RF at baseline and OLD and a trend between ACPA at baseline and RLD at 15 years was observed We did not find an association between smoking and RLD, whereas a strong association between smoking and RA-ILD was found in a previous study defining RA-ILD by means of carbon monoxide transfer factor, FVC $<80 \%$ pred and perfusion abnormality. ${ }^{12}$ Smoking, especially current smoking, was strongly associated with OLD, an observation previously observed in both the general population ${ }^{25} 26$ and RA population. ${ }^{10}$

Only a few other studies have investigated the association between demographic and clinical factors and both OLD and RLD in the same IP/RA cohort. In one study, including 159 patients with RA without cardiovascular disease and with a mean disease duration of 10 years, breathlessness, BMI and prednisone use were predictors of RLD, and chronic cough, prior diagnosis of lung disease, gender, exercise, BMI, current smoking, RF positivity and prednisone use were predictors of OLD in a final multivariate regression model. ${ }^{10}$ In our study we found an association between breathlessness and OLD, but not with RLD in univariate regression analysis. The MRC respiratory symptom questionnaire used in the present study was developed to detect respiratory symptoms, a surrogate marker of chronic obstructive pulmonary disease (COPD), and thus our results seem valid. The association between oral steroids and abnormal lung function tests found in our study and in the previous study may be a reflection of worse disease activity or because they are prescribed because of their lung disease.

Pulmonary toxicity of methotrexate has been reported in a number of studies ${ }^{27-29}$ but not in other investigations. ${ }^{30}$ Since methotrexate is the DMARD of first choice in most countries, we also investigated the association between methotrexate use and lung function test abnormalities in this study. Cross-sectionally, we did not find worse lung function in those patients who used methotrexate in the total population, but a protective effect was found in those with RA. We also analysed the effect of methotrexate use since symptom onset on lung function test abnormalities (data not shown), and again no association was found. This may be due to channelling bias, by which methotrexate treatment is avoided or discontinued in those with respiratory problems. Alternatively, the lack of association could reflect the fact that methotrexate pulmonary toxicity leads to either a severe pneumonitis (in which case the patient may be lost to follow-up) or a self-limiting pneumonitis from which the patient fully recovers when methotrexate is discontinued.

A number of problems arise when comparing results of lung function disorders in RA populations based on spirometry tests, especially RLD between studies. In contrast to OLD for which generally accepted criteria are available (ie, $\mathrm{FEV}_{1}$ pred $\leq 80 \%$ and $\mathrm{FEV}_{1} / \mathrm{FVC}<0.70$ ), no internationally accepted pulmonary function test criteria exist for RLD. Furthermore, the comparison group varies between studies. In our study, patients with RLD and OLD were compared with patients with normal lung function defined as FVCpred $\geq 70 \%$ and $\mathrm{FEV}_{1}$ pred $\geq 80 \%$ at 15 years. It is, however, possible that some patients with OLD at 15 years had RLD at baseline and vice versa.

The second objective of this study was to compare the prevalence of an abnormal lung function test in patients with IP and the general population in Norfolk, UK. OLD occurred more frequently in patients with IP than in the general population after adjusting for smoking, as previously observed.' In another study the prevalence of pulmonary function abnormalities was also higher in non-smoking patients with RA compared with the general population. ${ }^{8}$ Although RA-ILD is the most common form of lung involvement in RA and we did find an association between worse disease and RLD in this study, RLD was not more evident in the IP population than in the general population. Since RLD, once clinically apparent, rather than OLD is especially associated with increased mortality in RA, ${ }^{12}$ it is possible that those patients with the most severe RLD did not survive to the 15-year follow-up (see online supplemental file for a description of underlying causes of death) or were no longer participating in the study.

This study has some limitations. RLD was not confirmed by any other test such as HRCT. ${ }^{31}{ }^{32}$ The findings regarding the comparison of an abnormal lung function test between the IP population and the general population should particularly be interpreted with caution. The protocol used to assess $\mathrm{FEV}_{1}$ and FVC differed between the two cohorts. In order to define patients with OLD and RLD we had to estimate the expected $\mathrm{FEV}_{1}$ and FVC based on equations derived from the English population. Although the estimated and actual reading of both $\mathrm{FEV}_{1}$ pred and FVCpred were strongly correlated, it is known that the prevalence of COPD may vary between areas in the $\mathrm{UK}^{33}$ The difference in the obtained $\mathrm{FEV}_{1}$ value was small but statistically significant and the difference in the percentage of patients with OLD between the two populations was relatively large. A slight change in the value may already define whether or not a person has OLD or RLD. This was noticeable when a few patients with IP who were identified as having OLD based on the actual measurement no longer had OLD when the $\mathrm{FEV}_{1}$ pred was calculated again for the comparison study, although a strong correlation existed between the obtained $\mathrm{FEV}_{1}$ pred and the calculated $\mathrm{FEV}_{1}$ pred. Clinically, it is therefore important to perform other diagnostic tests as well. Another limitation is that those patients with IP with, for example, cardiovascular diseases or who were frail were excluded from this study whereas no exclusion criteria were applied in the EPIC cohort. This may have led to an underestimate of the true difference in abnormal lung function tests between the IP population and the general population because of the increased likelihood of abnormal lung function tests in people with these comorbidities.

In conclusion, OLD (but not RLD) is more prevalent in an established IP population than in the general population. Functional disability is especially associated with RLD whereas smoking is associated with OLD. The latter finding and the known association between smoking and a poor disease prognosis - and the better prognosis in those who stopped-strengthens the importance of smoking cessation in patients with inflammatory arthritis.

Acknowledgements We would like to acknowledge the contribution of the local general practitioners and rheumatologists in Norwich in referring patients to the register, the NOAR metrologists in the collection of the clinical data and the database management team in Manchester.

Contributors All authors contributed to the design of the study, interpretation of the results and approval of final version of the manuscript.

Funding NOAR is funded by Arthritis Research UK (grant reference 17552).

Competing interests None.

Ethics approval Ethics approval was obtained from the Norwich local research ethics committee, UK.

Provenance and peer review Not commissioned; externally peer reviewed.

Open Access This is an Open Access article distributed in accordance with the Creative Commons Attribution Non Commercial (CC BY-NC 3.0) license, which permits others to distribute, remix, adapt, build upon this work non-commercially, and license their derivative works on different terms, provided the original work is properly cited and the use is non-commercial. See: http://creativecommons.org/licenses/by-nc/3.0/ 


\section{REFERENCES}

1. Nannini C, Ryu JH, Matteson EL. Lung disease in rheumatoid arthritis. Curr Opin Rheumatol 2008;20:340-6.

2. Turesson C, O'Fallon WM, Crowson CS, et al. Extra-articular disease manifestations in rheumatoid arthritis: incidence trends and risk factors over 46 years. Ann Rheum Dis 2003;62:722-7.

3. Bartels $\mathbf{C M}$, Bell $\mathrm{CL}$, Shinki $\mathrm{K}$, et al. Changing trends in serious extra-articular manifestations of rheumatoid arthritis among United State veterans over 20 years. Rheumatology (Oxford) 2010;49:1670-5.

4. Metafratzi ZM, Georgiadis AN, loannidou CV, et al. Pulmonary involvement in patients with early rheumatoid arthritis. Scand J Rheumatol 2007;36:338-44.

5. Koduri G, Norton S, Young A, et al. Interstitial lung disease has a poor prognosis in rheumatoid arthritis: results from an inception cohort. Rheumatology (Oxford) 2010;49:1483-9.

6. Naz SM, Symmons DP. Mortality in established rheumatoid arthritis. Best Pract Res Clin Rheumatol 2007;21:871-83.

7. Amital A, Shitrit D, Adir Y. The lung in rheumatoid arthritis. Presse Med 2011:40:e31-48

8. Fuld JP, Johnson MK, Cotton MM, et al. A longitudinal study of lung function in nonsmoking patients with rheumatoid arthritis. Chest 2003;124:1224-31.

9. Geddes DM, Webley M, Emerson PA. Airways obstruction in rheumatoid arthritis. Ann Rheum Dis 1979;38:222-5.

10. Pappas DA, Giles JT, Connors G, et al. Respiratory symptoms and disease characteristics as predictors of pulmonary function abnormalities in patients with rheumatoid arthritis: an observational cohort study. Arthritis Res Ther 2010;12:R104

11. Gabbay $\mathbf{E}$, Tarala $R$, Will $R$, et al. Interstitial lung disease in recent onset rheumatoid arthritis. Am J Respir Crit Care Med 1997;156:528-35.

12. Saag KG, Kolluri S, Koehnke RK, et al. Rheumatoid arthritis lung disease. Determinants of radiographic and physiologic abnormalities. Arthritis Rheum 1996;39:1711-19.

13. Charles PJ, Sweatman MC, Markwick JR, et al. HLA-B40: a marker for susceptibility to lung disease in rheumatoid arthritis. Dis Markers 1991;9:97-101.

14. Symmons DP, Barrett EM, Bankhead CR, et al. The incidence of rheumatoid arthritis in the United Kingdom: results from the Norfolk Arthritis Register. $\mathrm{Br} \mathrm{J}$ Rheumatol 1994;33:735-9.

15. Thomson W, Harrison B, Ollier B, et al. Quantifying the exact role of HLA-DRB1 alleles in susceptibility to inflammatory polyarthritis: results from a large, population-based study. Arthritis Rheum 1999;42:757-62.

16. Prevoo ML, van't Hof MA, Kuper HH, et al. Modified disease activity scores that include twenty-eight-joint counts. Development and validation in a prospective longitudinal study of patients with rheumatoid arthritis. Arthritis Rheum 1995;38:44-8.

17. Kirwan JR, Reeback JS. Stanford Health Assessment Questionnaire modified to assess disability in British patients with rheumatoid arthritis. Br J Rheumatol 1986;25:206-9.

18. Arnett FC, Edworthy SM, Bloch DA, et al. The American Rheumatism Association 1987 revised criteria for the classification of rheumatoid arthritis. Arthritis Rheum 1988;31:315-24
19. National Clinical Guideline Centre. Management of adults with chronic obstructive pulmonary disease in primary and secondary care: update guideline (draft). NICE, 2009. http://www.nice.org.uk/nicemedia/pdf/COPDDraftConsultationGuideline.pdf

20. Bestall JC, Paul EA, Garrod R, et al. Usefulness of the Medical Research Council (MRC) dyspnoea scale as a measure of disability in patients with chronic obstructive pulmonary disease. Thorax 1999;54:581-6.

21. Medical Research Council. Definition and classification of chronic bronchitis for clinical and epidemiological purposes. A report to the Medical Research Council by their Committee on the Aetiology of Chronic Bronchitis. Lancet 1965;1:775-9.

22. Day N, Oakes S, Luben R, et al. EPIC-Norfolk: study design and characteristics of the cohort. European Prospective Investigation of Cancer. Br J Cancer 1999;80 (Suppl 1):95-103.

23. McFadden E, Luben R, Wareham N, et al. How far can we explain the social class differential in respiratory function? A cross-sectional population study of 21,991 men and women from EPIC-Norfolk. Eur J Epidemiol 2009;24:193-201.

24. Falaschetti E, Laiho J, Primatesta P, et al. Prediction equations for normal and low lung function from the Health Survey for England. Eur Respir J 2004;23:456-63.

25. Purdy S, Griffin T, Salisbury C, et al. Emergency respiratory admissions: influence of practice, population and hospital factors. J Health Serv Res Policy 2011;16:133-40.

26. Shavit 0, Swern A, Dong 0, et al. Impact of smoking on asthma symptoms, healthcare resource use, and quality of life outcomes in adults with persistent asthma. Qual Life Res 2007;16:1555-65.

27. Kremer JM, Alarcon GS, Weinblatt ME, et al. Clinical, laboratory, radiographic, and histopathologic features of methotrexate-associated lung injury in patients with rheumatoid arthritis: a multicenter study with literature review. Arthritis Rheum 1997:40:1829-37

28. Salliot C, van der Heijde D. Long-term safety of methotrexate monotherapy in patients with rheumatoid arthritis: a systematic literature research. Ann Rheum Dis 2009;68:1100-4.

29. Verstappen SM, Bakker MF, Heurkens AH, et al. Adverse events and factors associated with toxicity in patients with early rheumatoid arthritis treated with methotrexate tight control therapy: the CAMERA study. Ann Rheum Dis 2010;69:1044-8.

30. Dawson JK, Graham DR, Desmond J, et al. Investigation of the chronic pulmonary effects of low-dose oral methotrexate in patients with rheumatoid arthritis: a prospective study incorporating HRCT scanning and pulmonary function tests. Rheumatology (Oxford) 2002;41:262-7.

31. Youssef AA, Machaly SA, El-Dosoky ME, et al. Respiratory symptoms in rheumatoid arthritis: relation to pulmonary abnormalities detected by high-resolution CT and pulmonary functional testing. Rheumatol Int 2012;32:1985-95.

32. Biederer J, Schnabel A, Muhle C, et al. Correlation between HRCT findings, pulmonary function tests and bronchoalveolar lavage cytology in interstitial lung disease associated with rheumatoid arthritis. Eur Radiol 2004;14:272-80.

33. Nacul L, Soljak M, Samarasundera E, et al. COPD in England: a comparison of expected, model-based prevalence and observed prevalence from general practice data. J Public Health (Oxf) 2011;33:108-16. 